\title{
FORMACIÓN A DISTANCIA Y RETOS ACTUALES EN LOS ROLES DOCENTES Y SU VINCULACIÓN CON LA EMPRESA: PROPUESTA Y CONTROVERSIAS
}

\section{DISTANCE LEARNING AND CURRENT CHALLENGES IN TEACHING ROLES AND THEIR LINK TO THE COMPANY: PROPOSITION AND CONTROVERSIES}

\author{
Manuel Fandos Igado; manuel.fandos@unir.net \\ Universidad Internacional de la Rioja \\ Jacobo Cano Escoriaza; jcano@unizar.es \\ Universidad de Zaragoza
}

\section{RESUMEN}

La discusión sobre el análisis de los roles de los docentes cobra especial relevancia en la formación a distancia y en el contexto de una sociedad en crisis, compleja y cambiante. Es preciso abordar un trabajo colaborativo entre empresas de formación $-y$, en particular desde el modelo no presencial- y el ámbito universitario. Profundizando en el proceso de tutorización y seguimiento individualizado en la formación del alumnado.

Se enfatiza la necesidad de armonizar la voluntad de ofrecer una formación individualizada y la precisa estandarización de los productos y servicios que permitan la supervivencia de las mismas, especialmente este contexto desde la metodología de estudio de caso seleccionando el modelo que ha implementado un grupo formación.

\section{PALABRAS CLAVE}

Formación abierta, empresas, tutorización, rol docente, procesos de calidad, TIC

\section{ABSTRACT}

The discussion about the analysis of the teaching roles gets especial importance in distance learning and in the context of a complex and changing society which is in crisis. A work of collaboration between teaching companies -particularly from the distance teaching model 
point of view- and the university sphere is needed. It goes more deeply in the tutoring process and the personal follow-up all along the student's learning process.

The necessity of balance between the will to offer a personalized training and the necessary standardization of products and services, which allow the survival of the educational institutions, is emphasised especially in a crisis context.

Taking the methodology of the chosen case, the model implemented by one formation.

\section{KEY WORDS}

Open learning, companies, tutorisation, teaching role, quality processes, ITC,

\section{LA FORMACIÓN ABIERTA UNA APUESTA POR UNA FORMACIÓN INDIVIDUALIZADA EN CONTEXTOS DE CAMBIO.}

En el contexto de una sociedad compleja y en crisis, con vertiginosos y permanentes cambios socio-políticos y económicos, no cabe duda que la formación ejerce un papel esencial. Dicha formación debe estar enfocada a preparar a los ciudadanos del futuro, en las mejores condiciones posibles, para ser protagonistas de dicha sociedad y entroncada y ligada directamente con el sector socioproductivo y el propio mundo laboral.

En este sentido, se torna vital la necesidad de superar ciertos encorsetamientos que pudieran derivarse de un enfoque formativo eminentemente presencial, sin flexibilidad para atender las crecientes necesidades de los usuarios. Las coordenadas espacio-temporales, en este sentido, debieran considerarse como oportunidades y no como amenazas o debilidades del propio sistema formativo.

Por otro lado, la vertebración del territorio se consigue en gran medida con la incorporación de las tecnologías de la información y la comunicación. Desde esta perspectiva, la aldea global es un marco amplio en el que, en todo caso, hay que considerar las costumbres culturales, para, una vez más, adaptar la formación al contexto más cercano al usuario, desde la distancia.

La necesaria y, en ocasiones, lejana flexibilidad horaria y conciliación de la vida personal supone un reto. Dicho reto, además de condición básica, es indispensable para abordar una apuesta formativa de calidad que responda a las exigencias diversas de los usuarios y del mercado.

En este sentido la equidad y la igualdad de oportunidades pueden ser conseguidas en la medida en que se disminuye la brecha digital. De esta forma, también las personas con algún tipo de discapacidad o de cualquier otro condicionante pueden aprovechar las ventajas y posibilidades de un proyecto formativo a distancia, teniendo como eje vertebral y nuclear la atención individualizada. 
La formación a distancia es un fenónemo creciente y de enorme actualidad, analizado desde diversas investigaciones que aportan líneas esenciales para atender a los usuarios desde esta perspectiva (Hannafin et al. 1999; Beverly, 2000; Garrison y Anderson, 2003; Pelegrín et al., 2004; García Areitio et al. 2007; Barberá, 2008; Casamayor et al. 2008) y también desde la formación continua (Tabuenca et al. 2013). Este esfuerzo, en todo caso, es inútil si la Administración no afronta directamente el reto de la formación en este contexto (Servicio Público de Empleo Estatal, 2006).

No cabe duda, que en el ámbito de la formación, el protagonismo tradicional ha correspondido históricamente a las universidades (Ardizzone y Rivoltella, 2003; Barajas et al., 2003; Moreno y Santiago, 2003; Martínez, 2004; Marcelo, 2006). Al respecto existen evidencias interesantes del trabajo colaborativo a nivel interuniversiatario para el diseño de materiales para la evaluación de la e-formación a través de rúbricas (Cabero y Rodríguez, 2013). De igual modo, el pensamiento docente es clave para la implementación de las tecnologías de la información y la comunicación (Gómez y Cano, 2011) así como la adquisición de las diferentes competencias en el ámbito formativo universitario (Fernández y Sanjuán, 2012) y la evaluación de las distintas plataformas a nivel de las universidades (Castaño, Jenaro y Flores, 2012).

Sin embargo, no parecería tener un enfoque muy realista considerar este protagonismo en la actualidad, sin tener en cuenta el avance en calidad y en diversificación de la oferta formativa por parte de otras empresas e instituciones que han entrado a complementar dicha función y, en cierta medida, a competir por una cuota de mercado existente dada la diversidad de necesidades $y$, en ocasiones, por la lentitud en la adaptación del propio sistema universitario. En el fondo, el desarrollo tecnológico en general y de la e-formación y el e-learning en particular ha permitido la aparición de un nuevo «mercado».

Nos parece relevante partir aquí de una concepción del e-learning como un aprendizaje basado en las tecnologías de la información y la comunicación con el que se favorece a cada individuo la consecución de las pertinentes competencias profesionales (Marcelo et al., 2002).

Por otro lado la apuesta por la formación individualizada por parte de las empresas de formación debe ser clara en los propios objetivos empresariales, se trata, al cabo de dar respuestas individuales a cada uno de los "clientes» de las mismas. Se escenifica así una dialéctica entre el proceso personalizado de apoyo al usuario y la necesaria estandarización del producto, considerando el propio balance de beneficios, el necesario control de la calidad y la participación en la mejora de los procesos de mejora continua y la creciente cualificación profesional de los propios formadores, con nuevas dimensiones en su desempeño.

Al respecto nos parece interesante analizar un estudio comparativo internacional sobre modelos estandarizados de evaluación y marcas de calidad de materiales educativos digitales (Domínguez y Redondo, 2006: 157 en Servicio Público de Empleo Estatal) en el que se destaca la importancia de la puesta en escena de una concreción certera de las necesidades de los usuarios y, por consiguiente, la implementación de unas guías de orientación para el autoaprendizaje, la creciente interactividad del material, la evaluación de 
los procesos, resultados e impacto y la concreción de los distintos procesos en todo el proceso formativo.

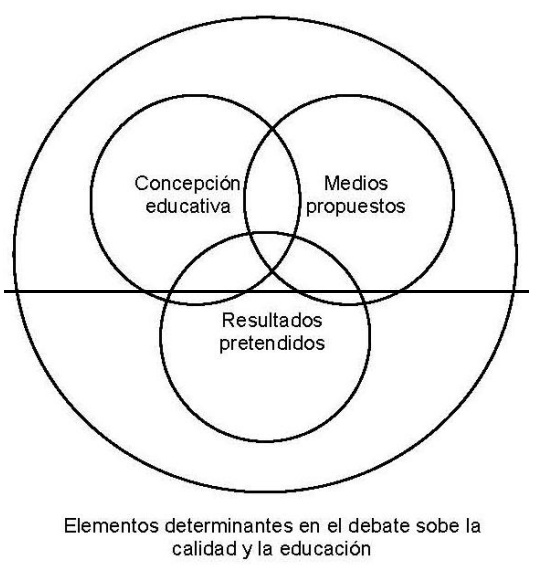

Gráfico 1. Modelos estandarizados de evaluación y marcas de calidad de materiales educativos digitales (Domínguez y Redondo, 2006).

\section{DIVERSIDAD DE ROLES EN EL PERFIL TUTORIAL EN LA FORMACIÓN A DISTANCIA: CLAVES PARA LA ACCIÓN}

La formación desempeña un papel clave en situaciones de cambio y de necesidad de cualificación profesional de los ciudadanos en un contexto de creciente demanda de actualización. La formación a distancia no se ha quedado atrás al considerar que la calidad debe ser un elemento vertebrador en toda oferta de este tipo (Kirkpatrick, 1999, Silvo, 2004, García, 2005, Martínez-Caro, 2005, Marcelo, 2008; Marcelo y Zapata, 2008).

Existen iniciativas (García, 2009:348-349) que van en la línea de ofrecer programas de calidad en educación a distancia en que aparecen elementos de gran relevancia. Todos tienen una importancia capital, pero nos centraremos en este apartado en el primero de ellos, donde hace hincapié en las características de los docentes.

Tabla 1. Elementos del Programa de Calidad en educación a distancia (García, 2009: 348-349)

Suficiencia científica, técnica y pedagógica de los docentes.

La formación continua y la incentivación.

La planificación.

El grado de satisfacción.

El liderazgo.

La participación.

La investigación y la evaluación.

La innovación.

Clarificación de los procesos de información y comunicación. 
Es indudable que desde la necesidad de entendimiento y sinergia de esfuerzos de todas las instituciones formativas, el papel del profesor-tutor es esencial. Éste tiene diversas funciones de gran importancia y que van desde el asesoramiento en la organización de los materiales al propio usuario (a nivel de contenido y estrategias de aprendizaje) al fomento de la competencia de aprender a aprender de forma autónoma, al tiempo que debe considerar la autoevaluación desde un enfoque exigente y realista.

Así pues, parece claro que algunas de las funciones del profesor-tutor en los diversos entornos virtuales son las de animar, mediar, seleccionar, facilitar, moderar... cualquiera que permita el intercambio entre él mismo y el alumno y/o los alumnos entre sí, naturalmente con una intencionalidad educativa.

Más aún, el logro de los aprendizajes, la consecución de las metas fijadas con cada alumno realizados como consecuencia de un contrato entre el usuario y el propio responsable directo de la formación, es uno de los indicadores esenciales para seguir el proceso tutorial de forma individualizada hacia cada uno de los participantes.

Pero no sólo debe valorarse dicho logro, sino que también es importante valorar la calidad en el proceso de adquisición de dichos aprendizajes, considerando que éste se realiza en un contexto de formación a lo largo de la vida.

Las condiciones personales y profesionales de aquellos usuarios que desean o tienen necesidad de aumentar su formación, pueden ser muy complejas y peculiares. En este sentido, es preciso dotar de la flexibilidad necesaria el correspondiente itinerario formativo. Es aquí donde las instituciones formativas deben realizar un esfuerzo importante para ofrecer la tutorización u orientación de calidad que se demanda.

El tutor debe mostrar una notable capacidad de comunicación, considerando todos los factores que intervienen en la comunicación, tanto la oral, como la escrita y la gestual. Es preciso subrayar y también el dominio de técnicas de comunicación, gestión y manejo del ámbito telemático y audiovisual.

Las funciones de los tutores en este tipo de entornos son diversas tal y como venimos señalando y coincidimos en destacar en la siguiente tabla, en la línea de García (2009: 276), las referidas a la facilitación del aprendizaje. En este sentido, remarcamos la necesidad de desarrollar estrategias, proponer recursos e incorporar la incentivación de la motivación de las personas en los correspondientes procesos de formación:

Tabla 2. Funciones de los tutores virtuales según García (2009)

Diseñador y gestor del proceso.

Orientador personal.

Proveedor de información y recursos.

Generador de ambiente propicio y dinamizador de grupo.

Motivador y facilitador de aprendizaje.

Supervisor y evaluador. 
Valorando una perspectiva complementaria, Ardizzone y Rivoltella (2004: 131) desde su colaboración con el Centro para la Educación Permanente y a Distancia de Milán (CEP@D), exponen las funciones tutoriales referidas más específicamente al ámbito universitario.

Tabla 3. Funciones de los tutores virtuales universitarios según (Ardizzone y Rivoltella 2004)

Coplanificar, cogestionar y resolver problemas derivados del proceso de formación a distancia.

Comprender los contextos diversos en el ámbito de formación.

Conocer los instrumentos pertinentes a nivel ténico.

Leer los target.

Favorecer la interacción y la posibilidad de colaboración.

La perspectiva del aprovechamiento de recursos y posibilidades en el proceso de acción tutorial es el que se vislumbra de las aportaciones de IPSE (2000) y recogido en Barberá (2008: 51) remarcando las siguientes:

Tabla 4. Adaptación de las funciones de los tutores virtuales según IPSE (2000)

Planificación de actuaciones según necesidades de estudiantes.

Consideración del aprendizaje a lo largo de la vida y las ofertas cursadas por los estudiantes.

Revisión y evaluación continua para la mejora de la calidad.

Descripción de resultados de aprendizaje progresivos.

Adaptación a los diferentes estilos de aprendizaje.

Aprovechamiento de la formación institucional y búsqueda de sinergia con los recursos humanos existentes para la eficiencia en la utilización de recursos materiales.

Respecto al papel de los profesionales que diseñan los materiales y de los tutores, siguiendo a Barberá (2006) es preciso valorar la importancia de la claridad en el contenido, la secuenciación en el proceso de enseñanza-aprendizaje y el grado de entendimiento. La dinamización de dicha acción formativa, la facilitación del aprendizaje, la motivación al estudiante y la evaluación del proceso formativo son esenciales que el tutor las tenga muy en cuenta para adaptarse y considerar cada una de estas variables.

El profesional que se encarga de diseñar los materiales debe implementar las pertinentes actuaciones cuyo fin es que el aprendiz adquiera las competencias propuestas, teniendo en cuenta en todo caso el ritmo de aprendizaje diverso, matizados por los condicionantes personales (Casamayor et al., 2008). 


\section{ANÁLISIS DE CASO DE LA FORMACIÓN A DISTANCIA: DILEMAS Y OPORTUNIDADES}

Es importante señalar que el e-learning como concepto tiene una serie diversa de acepciones tales como la psicología y pedagogía (teorías del aprendizaje y didáctica), la economía, la comunicación, el comercio, la tecnología o la ingeniería, en su vertiente de organización de procesos (Barberá, 2008).

Traemos aquí una experiencia que aborda esta cuestión tan poliédrica un grupo empresarial referente en el sector de la formación, concretamente en la formación abierta, para ver cómo ha afrontado este problema y analizar algunas de sus actuaciones y logros. Esta empresa, como otras del sector, se encontró al poco tiempo de comienzo de su actividad ante estas dos variables que citábamos arriba, por un lado la exigencia de la personalización o individualización del servicio y, por otro, la necesidad de la estandarización de esos productos, servicios y procesos. En el fondo se trata de una compatibilización imprescindible para garantizar la continuidad de la compañía (si se quiere del propio negocio).

Dos variables, que, sin duda presentan una dificultad conciliadora ¿cómo superar la posible incompatibilidad entre la «individualización» de la educación y la «estandarización» en los procesos, modelos y servicios que la compañía ofrece? (Aguaded y Fandos, 2008).

En realidad, consciente o inconscientemente, el modelo de "formación abierta» de muchas de las empresas de formación, sobre todo el de aquellas que ponen el acento en la "formación abierta» coinciden con Coathen (2003) y Mars (2003) cuando definen el «blended learning» como un modo de aprender que combina la enseñanza presencial con la tecnología no presencial, porque la idea esencial es la de considerar que la clave del éxito está en cualquier posible combinación de todos los medios posibles que faciliten el aprendizaje y resuelva los problemas específicos de cada aprendiz.

Por otro lado, el aprendizaje es un hecho individual y personal, y la tarea fundamental de las empresas de formación no es incidir sobre el aprendizaje, de hecho no puede hacerlo. Se trata de incidir en el acompañamiento, en el asesoramiento, en facilitar los medios (materiales e inmateriales) que a cada uno le permitan interiorizar, aprender, y generalizar aquello que necesita. Del mismo modo, desde una óptica empresarial, al menos, el modelo y la calidad del mismo, se basan en ofrecer a cada uno lo que necesita y cuando lo necesita.

Queda claro, por lo tanto, que es el usuario de los productos y servicios del centro y no el profesor el que ha hacer girar todo el sistema a su alrededor.

Para hacer pivotar todo el conjunto de servicios y productos que la compañía ofrece a sus (alumnos - clientes) esta empresa proponer al usuario un itinerario adaptado (que debe aceptar) para alcanzar su objetivo (dado que, en muchos casos, la preparación no es más que un camino que conduce a un objetivo, y que ésta no es el objetivo en sí mismo).

Así la meta prefijada queda clara desde el principio y, a medida que cada uno va alcanzando las distintas fases y los hitos señalados el usuario va teniendo acceso a nuevos servicios, apoyos y materiales. Es un primer paso importante que permite la individualización y la personalización, vinculada al propio esfuerzo del alumno. 
El trabajo y el proceso de aprendizaje es individual, pero periódicamente el sujeto tiene que demostrar que su parte del trabajo se está cumpliendo. Para demostrarlo tiene que pasar por unos hitos de control, que permiten tanto la autoevaluación como la heteroevaluación y, en su caso, la reconducción de los planes individuales de cada uno. Los profesionales que ejercen de tutores ( $y$ entrenadores en el caso de esta compañía) son fundamentales en este plan de desarrollo individual (Casamayor et al., 2008).

La puesta en funcionamiento de estos check points hizo que esta empresa, en su origen netamente de formación a distancia, sufriera una profunda metamorfosis que desembocó en una empresa de "formación abierta y de entrenamiento». Quizás ahí radica uno de sus grandes logros y secretos.

Y en esta misma línea de centrarse en el servicio al cliente y de facilitar esencialmente el trabajo individual que genere el aprendizaje particular, esta compañía ha entendido que es esencial hacer importantes esfuerzos inversores en productos, sedes y tecnologías, entendidos como un vehículo que permite un mejor servicio, por lo tanto, permite mejorar los resultados.

Unos resultados tanto desde el punto de vista del éxito del alumno, como desde el punto de vista de la satisfacción del mismo que comprometen contractualmente con sus alumnos de manera importante a la empresa. Este compromiso con el resultado final es tan relevante que correlaciona de manera sustantiva con al salario del equipo de personas que brindan la atención y los servicios a los usuarios.

Por lo tanto, la disyuntiva que planteábamos al principio que parecía insalvable por un lado la necesidad de personalización e individualización de la intervención docente, y por otro lado la necesidad de la estandarización del servicio, -entre otras razones, para abaratar costes y garantizar la supervivencia de la compañía-, este modelo formativo ha conseguido armonizarla con:

- La propuesta de un método que parte de una planificación individualizada, que ofrece unos productos y servicios graduados, en función de los resultados acreditados y sistemáticamente medidos.

- Un notable esfuerzo inversor y de desarrollo tecnológico.

- La implicación directa del alumno-cliente, llevándole a adquirir un compromiso de trabajo individual personal, que aunque goce de todo tipo de acompañamientos y ayudas es personal de intransferible del estudiante.

- Un compromiso con los resultados de aquellos que se implican, hasta el punto de devolver el dinero al cliente si no consigue su objetivo y aumentar o reducir las retribuciones de quienes hayan participado en el acompañamiento y tutorización de cada persona.

En definitiva la disyuntiva de partida se puede superar cuando hay un empeño en poner el acento en alcanzar el resultado de quien estudia con una intencionalidad que va más allá del propio estudio en sí mismo. Tal vez, una de las claves del éxito de esta compañía, haya sido entender que para buena parte de sus estudiantes-clientes el estudio no es una meta en sí misma, sino un camino que les puede conducir a un objetivo mucho más deseado, y el 
acompañamiento ha de hacerse hasta la consecución de la misma, no sólo durante una parte del proceso.

\section{CONCLUSIONES}

El proceso de formación a distancia adquiere una gran relevancia el marco de la sociedad compleja y del conocimiento en la que estamos inmersos. Resulta acuciante la necesidad de implementar estrategias para el desarrollo de las diferentes competencias transversales, generales y específicas para mejorar, entre otras cosas la empleabiliad de los estudiantes; por lo tanto, la formación va asociada a un objetivo que transciende le mero hecho de estudiar o cualificarse.

En este proceso, intervienen diferentes actores que cobran un creciente protagonismo. Por una parte, la propia Universidad desarrollando su papel en la formación a lo largo de la vida, y teniendo en este momento diversas carencias en cuanto a falta de flexibilización para adaptarse a las diferentes necesidades formativas. En este sentido hemos remarcado distintas experiencias y trabajos de investigación en torno a la evaluación de la adquisición de diferentes competencias en el marco de las tecnologías de la información y la comunicación así como en la propuesta de evaluación de plataformas virtuales (Castaño, Jenaro y Flores, 2012) y diseño de instrumentos como rúbricas (Cabero y Rodríguez, 2013).

En todo caso se torna necesario implementar sistemas de gobernanza y gestión en la institución universitaria que mire más de cerca y tienda puentes con el tejido empresarial, en los diferentes niveles autonómico, nacional e internacional.

Dicho tejido empresarial, junto con los sindicatos y los diferentes agentes que adquieren protagonismo en la formación, pueden jugar un papel esencial en la medida en que implementan estrategias adaptativas al perfil diverso y cambiante del candidato a la formación, entendida ésta como un proceso que dura toda la vida y adquiriendo una importancia capital el papel del tutor-profesor como mediador y guía en el propio proceso de aprendizaje.

En un contexto de crisis globalizada a nivel económico esencialmente, el e-learning puede ser un potencial importante para desarrollar una formación de calidad, al tiempo que posibilite una mayor eficiencia en la gestión de los diferentes recursos humanos y materiales (también espacio-temporales). Las infraestructuras necesarias para incorporar en un primer momento deben estar dentro del plan estratégico de desarrollo empresarial (incluso universitario). En este sentido, el análisis del caso de la compañía referida puede ser un punto de referencia en la gestión de los servicios y su implantación tanto pautada (condicionada), expansiva y diversificada, como en la implicación de los agentes que intervienen en los procesos vinculando parte de sus retribuciones a los éxitos de sus tutelados -siempre y cuando estos hayan cumplido con los compromisos adquiridos previamente-. 
Por otro lado, el factor tiempo, escaso y preciado para los diferentes usuarios, es otro de los elementos que las instituciones formativas deben considerar de una manera prioritaria.

La formación a distancia, ineludiblemente, debe albergar un sistema de calidad si no adaptado, sí relacionado con la misión, visión y valores del tejido empresarial -y de cada empresa de forma concreta- para mejorar el sistema de establecimiento de protocolos pertinentes adaptados a las necesidades de los usuarios y de los objetivos de cada centro o institución formativa.

Los contenidos a desarrollar, los recursos tecnológicos específicos deben basarse en un modelo pedagógico que considere las necesidades formativas existentes. La evaluación de la calidad de la misma, así como su grado de implicación y vinculación con la futura práctica profesional son dos de los elementos esenciales en toda oferta formativa de calidad.

Los gestores de las acciones formativas, así como el rol de los tutores, son agentes clave para superar las barreras culturales, tecnológicas y del conocimiento de los diferentes perfiles de usuarios, adaptándose lo más posible a las necesidades manifestadas para mejorar el proceso de aprendizaje de forma personalizado.

El aprendizaje negociado, combinado, colaborativo se puede complementar con aquel desarrollado a través del propio autoaprendizaje. En cualquier caso, es clave considerar el papel de la motivación en el contexto en el que se desenvuelven los diferentes usuarios.

A nivel formativo y empresarial, se vislumbra un interesante y expansivo campo de actuación en todo lo referente a desarrollar dispositivos inalámbricos que permitan el aprendizaje flexibles asíncrono, tal y como se recoge con el mobile-learning, incluso el ulearning. Resulta clave de cara al futuro mejorar la operatividad real para superar las limitaciones tecnológicas en las aplicaciones didácticas utilizadas tradicionalmente.

La creación de redes de apoyo, a nivel empresarial y con carácter interdisciplinar, es uno de los aspectos diferenciadores entre aquellas instituciones formativas más robustas en relación con otras con una visión estratégica menos desarrollada.

Finalmente, aunque de forma más o menos tangencial, es necesario avanzar en un adecuado marco jurídico que posibilite, por una parte, la clarificación de los aspectos relacionados con los derechos de autor de los materiales didácticos ofrecidos a los participantes de este tipo de formación, y por otra, un auténtico reconocimiento institucional, administrativo, social y laboral de los aprendizajes logrados en este tipo de contextos en el que, cada vez más, intervienen tantos agentes sociales, colectivos, empresas e instituciones.

\section{REFERENCIAS}

AGUADED, I. y FANDOS, M. (2008). Blended Learning: The Key to Success in a Training Company. ITDL. International Journal of Instructional Technology and Distance Learning, 5, $8 . \quad$ Recuperado el 22/05/2013 de http://itdl.org/Journal/Aug 08/article04.htm

ARDIZZONE, P. y RIVOLTELLA, P.C. (2004). Didáctica para e-learning. Métodos e instrumentos para la innovación de la enseñanza universitaria. Málaga: Aljibe. 
BARAJAS, M. et al. (2003). La tecnología educativa en la enseñanza superior: entornos virtuales de enseñanza. Madrid: McGraw-Hill.

BABERÁ, E. (COORD.) (2006). Educación abierta y a distancia. Barcelona: UOC.

BARBERÁ, E. (2008). Aprender e-learning. Barcelona: Paidós.

BARTOLOMÉ, A. (2004). Blended learning conceptos básicos. Blended learning. Pixel Bit. Revista de Medios y Educación. 23, 7-20.

BEVERLY, A. (2000). Intructional and cognitive impacts of Web-Based Education. USA: Idea Group Publishing, Hershey.

CABERO, J. y RODRÍGUEZ, M. (2013). La utilización de la rúbrica en el diseño de materiales para la e-formación. EDUTEC Revista Electrónica de Tecnología Educativa 43. Recuperado el 22/05/2013 de http://edutec.rediris.es/Revelec2/Revelec43/utilziacion rubrica diseno materiales e-formacion.html

CASAMAYOR, G. (Coord.) et al. (2008). La formación online. Una mirada integral sobre el elearning, b-learning. Barcelona: Graó.

CASTAÑO, R.; JENARO, C. y FLORES, N. (2012). Análisis DAFO de la utilidad de las plataformas de formación online para el entrenamiento en competencias de estudiantes universitarios.EDUTEC, Revista Electrónica de Tecnología Educativa, 42. Recuperado el 23/05/2013 de http://edutec.rediris.es/Revelec2/Revelec42/analisis DAFO utilidad plataformas formacion online competencias.html

COATHEN, N. (2003). Blended e-learning. Educaweb. 69. Octubre. [Artículo en línea]. Recuperado el 22/05/2013 de http://www.educaweb.com/esp/servicios/monografico/formacionvirtual/1181076.asp

FERNÁNDEZ, M.D. y SANJUÁN, M. del M. (2012).Entornos virtuales de aprendizaje: ¿Una ocasión para que nuestros estudiantes universitarios adquieran competencias profesionales? EDUTEC, Revista Electrónica de Tecnología Educativa, 42. Recuperado el 20/05/2013 de http://edutec.rediris.es/Revelec2/Revelec42/entornos virtuales aprendizaje ocasion estudiantes aquieran competencias profesionales.html

GARCÍA ARETIO, L. et al. (2007). De la educación a distancia a la educación virtual. Barcelona: Ariel.

GARCÍA ARETIO, L. (2009). ¿Por qué va ganando la educación a distancia? Madrid: UNED.

GARCÍA HOZ, V. (1988). La práctica de la educación personalizada. Vol 6. del Tratado de educación personalizada, GARCÍA HOZ, V. (director). Madrid: Rialp.

GARCÍA MARTÍNEZ, F. (2005). La calidad de la educación virtual a examen. Granada: Grupo Editorial Universitario.

GARRISON, D.R. y ANDERSON, T. (2003). E-Learning in the 21st Century: A Framework for Research and Practice. New York: Routledge.

GÓMEZ, J. y CANO, J. (2011): El pensamiento docente y su influencia en la implantación de las Tecnologías de la Información y la Comunicación en el aula: desafíos y oportunidades. Contextos educativos $14,67-83$ 
HANNAFIN, M., LAND, S. y OLIVER, K. (1999). Entornos de aprendizaje abiertos: fundamentos, métodos y modelos. In C. Reigeluth (Ed.), Diseño de la instrucción. Teorías y modelos. Un nuevo paradigma de la teoría de la instrucción. Parte I. (pp. 125-152). Madrid: Aula XXI Santillana.

INDIANA PARTNERSHIP FOR STATEWIDE EDUCATION (IPSE), Guiding Principles for Faculty in Distance Learning (2000). Recuperado el 22/05/2013 de http://www.ihets.org/learntech/principles-guidelines.pdf

KIRPATRICK, D. (1999). Evaluación de acciones formativas. Los cuatro niveles. Barcelona: PISE.

MARCELO, C. et al. (2002). E-learning teleformación. Diseño, desarrollo y evaluación de la formación a través de Internet. Barcelona: Gestión 2000.

MARCELO, C. (COORD.) (2006). Prácticas de e-learning. Barcelona: Octaedro.

MARCELO, C. (2008). Cuestionario para la evaluación. Metodología e indicadores. RED, Revista de Educación a Distancia. Número monográfico VII.- Recuperado el 22/05/2013 de http://www.um.es/ead/red/M7/

MARCELO, C. y ZAPATA, M. (2008). Cuestionario para la evaluación: "Evaluación de la calidad para programas completos de formación docente a través de estrategias de aprendizaje abierto y a distancia". Metodología de uso y descripción de indicadores. RED, Revista de Educación a Distancia. Número monográfico VII.- 30 de Diciembre de 2008. Recuperado el 22/05/2013 de http://www.um.es/ead/red/M7/

MARS, G.; MCFADDEN, A. y PRICE, B. (2003). Blended Instruction: adapting conventional instruction for large classes, en Online Journal of Distance Learning Administration. № 4. [Artículo en línea]. Recuperado el 22/05/2013 de http://www.westga.edu/\%7Edistance/ojdla/winter64/marsh64.htm

MARTínEZ-CARO, E. (2005). La mejora de la calidad en la educación mediante entornos virtuales de aprendizaje. Tesis Doctoral. Universidad Politécnica de Cartagena.

MARTíNEZ, F.J. (2004). Enseñanza virtual y e-learning para profesores universitarios principiantes. Huelva: Martínez López editores.

MORENO, F. y SANTIAGO, R. (2003). Formación online. Guía para profesores universitarios. La Rioja: Universidad de La Rioja.

PELEGRÍN, C. (2004). E-learning: las mejores prácticas en España. Madrid: Pearson Educación.

SERVICIO PÚBLICO DE EMPLEO ESTATAL (2006). La formación sin distancia. Madrid: Servicio Público de empleo estatal.

SILVO, J. (2004). Reflexiones sobre la calidad en la educación virtual. La Educ@cion, 139-140, $1-9$.

TABUENCA, B.; TERNIER, S. y SPECHT, M. (2013). Patrones cotidianos en estudiantes de formación continua para la creación de ecologías de aprendizaje. Revista de Educación a distancia XII, 37. Recuperado el 22/05/2013 de http://www.um.es/ead/red/37/ 
Para citar este artículo:

FANDOS, M. \& CANO, J. (2013). Formación a distancia y retos actuales en los roles docentes y su vinculación con la empresa: propuesta y controversias. EDUTEC, Revista Electrónica de Tecnología Educativa, 45.4 ecuperado $\quad \mathrm{dd} / \mathrm{mm} / \mathrm{aa}$ de http://edutec.rediris.es/Revelec2/Revelec45/formacion distancia roles docentes vinculacion empr esa.html 